\title{
Viscosity effects on local scour around vertical structures in clear-water conditions.
}

\author{
Costantino Manes ${ }^{1,}$, Francesco Coscarella ${ }^{2}$, Ashley Rogers ${ }^{3}$, and Roberto Gaudio ${ }^{2}$ \\ ${ }^{1}$ Department of Environment, Land, and Infrastructure Engineering, Politecnico di Torino, Corso Duca \\ degli Abruzzi, 24, 10129, Torino, Italy. \\ ${ }^{2}$ Dipartimento di Ingegneria Civile, Università della Calabria, 87036, Rende, Italy \\ ${ }^{3}$ Faculty of Engineering and the Environment, University of Southampton, SO171BJ, Southampton, \\ UK.
}

\begin{abstract}
Local scour represents the erosion process that occurs at the base of hydraulic structures overlying sediment beds. Horseshoe vortices forming at the bed-structure junction are the main responsible for sediment removal and dictate erosion rates as well as the maximum erosion depth resulting from a significant flow event. In steady-flow conditions this is often referred to as the equilibrium scour depth, which, for many hydraulic structures, represents a key parameter for foundation-design and risk-assessment purposes. The equilibrium scour depth has been investigated for decades and many predictive formulae have been developed following the classical empirical approach, whereby numerous experimental datasets are used to isolate and identify the influence of non-dimensional groups emerging from dimensional analysis. Within this context, the influence of obstacle Reynolds numbers, and consequently of viscous forces, has always been neglected because of the large $R e$ values normally encountered in engineering and laboratory conditions. The present paper demonstrates that this assumption is largely incorrect especially for beds made of sand or finer material. The theoretical analysis presented in Manes and Brocchini ([1]) is herein extended to include viscosity effects and investigate their importance on equilibrium scour depths forming around obstacles resembling bridge piers.
\end{abstract}

\section{Introduction}

When a turbulent flow impinges a solid body lying over a sedimentary bed, a concentrated system of vortices forms at the base of the obstacle, which promotes sediment erosion and hence the formation of a scour hole. This phenomenon is commonly referred to as local scouring and is of paramount importance for the design and risk assessment of hydraulic structures.

The main core of research on local scour has been traditionally developed within the context of bridge hydraulics and the prediction of scour depths around piers and abutments subjected to flood events. Predictive formulae of local scour have long been developed following an

* Corresponding author: costantino.manes@polito.it 
empirical approach whereby numerous experimental datasets are used to isolate and identify the influence of non-dimensional groups emerging from dimensional analysis.

Let us consider the simplest case of an open-channel flow with a steady depth-averaged velocity $U$, flow depth $Y$ and a rectangular cross-section of width $B$, flowing over a sediment bed with uniform diameter $d$ and impinging a cylindrical structure piercing the free-surface of the flow and having a characteristic width $a$. Starting from an initial flat bed, the scour hole will develop until it reaches a steady state. The depth of the scour hole at such equilibrium conditions is referred to as the equilibrium scour depth $\left(y_{s e}\right)$ and is measured as the maximum depth of the scour hole measured from the undisturbed bed level. $y_{s e}$ is routinely used as a design parameter for foundation depth or, in risk assessment procedures, as a proxy to estimate bridge exposure to local scour with respect to extreme hydrological events associated with an arbitrarily chosen return period ([2]).

In the present paper we consider local scour under clear water conditions, whereby sediment transport does not occur upstream of the cylinder. We also consider isolated obstacle conditions so that local scour is not affected by the presence of lateral boundaries (i.e. $B>>a$ ) or other obstacles. Upon application of dimensional analysis and the Buckingham theorem it can be shown that the non-dimensional equilibrium scour-depth depends on the following non-dimensional groups (see e.g. [3]):

$$
\frac{y_{s e}}{a}=\phi\left\{\frac{a}{B}, \frac{Y}{a}, F r_{a}, \frac{a}{d}, \frac{U}{V_{c}}, \operatorname{Re}, \frac{\rho_{s}}{\rho}\right\}
$$

where, $F r_{a}=U / \sqrt{g a}$ is the cylinder Froude number, $R e=U a / v$ is the obstacle Reynolds number ( $v$ being the kinematic fluid viscosity), $\rho_{s}$ is the sediment density, $\rho$ is the fluid density and $V_{c}$ is the critical velocity of incipient sediment motion in the undisturbed channel. As pointed out in [1] (hereafter referred to as MB15), the non-dimensional groups listed above stem naturally from the application of the Buckingham theorem except for $U / V_{c}$ which is artificially introduced to discern between clear-water $\left(U / V_{c}<1\right)$ and live-bed $\left(U / V_{c}>1\right)$ conditions, with the latter implying sediment transport occurring not only in proximity of the obstacle but also in the undisturbed flow upstream.

In the literature about local scour, there is general consensus in considering the effects of $R e$ on $y_{s e} / a$ as negligible (see e.g. [4]). The commonly-employed argument in support of this hypothesis is based on the fact that, in both laboratory and field conditions, Re reaches very high values implying fully-developed turbulent flow regimes and consequently negligible viscosity effects. This view has been challenged only by a handful of studies. In [5], Ettema et al. acknowledged some $R e$ influence on $y_{s e} / a$ although not strictly in terms of viscosity effects but rather in terms of large-scale turbulence intensity. In [6], Lança et al. carried out an original set of experiments where viscosity effects where isolated and quantified by means of a dimensionless sediment diameter defined as $d_{*}=\sqrt[3]{g\left(\frac{\rho_{s}}{\rho}-1\right) \frac{d^{3}}{v^{2}}}$ (these authors use also an unconventional dimensional-analysis strategy to quantify viscosity effects, which involve dimensionless groups that differ from those reported in Equation 1). The analysis of experimental data revealed that $y_{s e} / a$ decreases with increasing $d_{*}$ and corroborated the results from some early studies carried out in [7, 8]. Finally, MB15 demonstrated that viscosity is indeed important as it may contribute to dictate the condition of incipient sediment motion within the scour hole. This effect is clearly negligible if the flow within the scour hole is in the fully-rough turbulent regime. However, following Shields and other studies related to sediment incipient motion (see, e.g., [9]), the fully-rough regime is unlikely 
to occur in beds made of sand or finer material, which, nevertheless, are very common in laboratory studies and real full-scale engineering problems.

In the present paper we extend the theoretical framework presented by MB15 to include viscosity effects (section 2). This general framework of analysis is then assessed with the aid of experimental data taken from the literature (section 3). Section 4 presents concluding remarks.

\section{Theoretical framework}

MB15 developed a phenomenological theory for the prediction of local scour around piers, which is valid if the flow within the scour hole is in the fully-rough regime. In this section, first we provide a summary of the key aspects of the MB15's theory, then we extend such theory to include viscosity effects.

In simple terms, MB15 hypothesized that the turbulent shear stress acting in the deepest point of the scour hole scales as

$$
\tau \sim \rho u_{d} V,
$$

where $V$ is the characteristic velocity of the horseshoe vortex forming at the base of the pier, which is largely considered the main responsible for sediment entrainment in local scour ([3]); $u_{d}$ is the characteristic velocity of eddies scaling with the sediment diameter. Following Kolmogorov's theory of turbulence and assuming such eddies belong to the inertial scales of turbulence, then

$$
u_{d} \sim V\left(\frac{d}{s}\right)^{1 / 3}
$$

where $S$ is the characteristic length scale of the horseshoe vortex, which is notoriously buried within the scour hole and therefore can be taken as a proxy for the scour depth. By means of energetic considerations, MB15 derived the following scaling for $V$

$$
V \sim U\left(\frac{C_{d} a}{S}\right)^{1 / 3}
$$

where $C_{d}$ is the drag coefficient of the structure.

From equations (2)-(4), it can be inferred that the shear stress scales as

$$
\tau \sim \rho U^{2}\left(\frac{C_{d} a}{S}\right)^{2 / 3}\left(\frac{d}{S}\right)^{1 / 3}
$$

Following Shields, at equilibrium conditions, $\tau \approx \tau_{c} \approx g d\left(\rho_{s}-\rho\right)$ and $S$ approaches $y_{s e}$. Hence,

$$
\frac{y_{s e} g}{U^{2}} \sim \frac{\rho}{\rho_{s}-\rho} C_{d}^{2 / 3}\left(\frac{a}{d}\right)^{2 / 3}
$$

which, as far as clear-water scour is concerned, represents MB15's final result. 
This theory is now extended to include viscosity effects. Towards this end, it is necessary to relax the assumption of fully-rough conditions in the formulation of $\tau$ (i.e. add a viscous component) and include a dependence on viscosity of the critical shear stress $\tau_{c}$. In the classical theory of incipient motion, the latter is usually done through the particle Reynolds number, which is defined as the ratio between the characteristic size of sediments and the viscous length scale. Here, consistently with the phenomenological approach proposed by MB15, the viscous length scale is replaced by the Kolmogorov length scale $\eta$ so that

$$
\frac{\tau_{c}}{g d\left(\rho_{s}-\rho\right)}=f\left(\frac{d}{\eta}\right)
$$

where, following MB15, $\eta$ is defined as

$$
\eta \sim\left(\frac{v^{3} S}{V^{3}}\right)^{1 / 4} \sim\left(\frac{v^{3} y_{s e}}{V^{3}}\right)^{1 / 4}
$$

and $f\left(\frac{d}{\eta}\right)$ is a function that can be found empirically from experimental data. The function $f\left(\frac{d}{\eta}\right)$ can be interpreted as a Shields curve for local scour phenomena.

A more challenging task is to include the effects of viscosity in $\tau$. Towards this end, however, it is possible to exploit the properties of turbulence within the inertial and the dissipative range, which are considered to be universal. According to Batchelor, the second order structure function $D(r)$ in such regimes can be empirically expressed as

$$
\frac{D(r)}{v_{\eta}^{2}}=\frac{r^{2} / 3 \eta^{2}}{\left[1+(1 / 3 b)^{3 / 2}(r / \eta)^{2}\right]^{1-\zeta / 2}}
$$

where $v_{\eta}$ is the so-called Kolmogorov velocity; $\zeta=2 / 3$; and $b=5.834$. This function is widely recognised to reproduce turbulence phenomena within the inertial-dissipative range very accurately and can be applied with confidence (see e.g. [10]). Equation (9) will be used as a model to capture the characteristic velocity of eddies at a relevant characteristic length scale $l$, i.e.,

$$
u_{l}=\sqrt{D(l)}
$$

In transitionally-rough flows over rough boundaries the characteristic length scale of nearwall eddies that contributes the most to momentum transfer is (see [11])

$$
l=d+b \eta
$$

where $b=5 \div 10$. Equation (10) essentially states that such eddies scale with the sediment diameter plus the thickness of the viscous sublayer (i.e. $5 \eta$ ) that forms in proximity of the sediment grains. If $\eta$ goes very small (i.e. $R e$ increases significantly), momentum transfer is dominated by roughness only and the fully-rough condition is re-established.

It follows that the shear stress exerted by a flow on a transitionally-rough surface can be generalized as

$$
\tau \sim \rho u_{l} V=\rho \sqrt{D(l)} V .
$$


At equilibrium conditions, $\tau$ and $S$ approach $\tau_{c}$ and $y_{s e}$, respectively. Hence, combining equations (4), (7) and (12) one obtains

$$
\tau_{c} \sim \rho \sqrt{D(l)} U\left(\frac{C_{d} a}{y_{s e}}\right)^{1 / 3}=f\left(\frac{d}{\eta}\right)\left(\rho_{s}-\rho\right) g d,
$$

and, finally

$$
\frac{\tau_{c}}{\left(\rho_{s}-\rho\right) g d}=\frac{\rho}{\rho_{s}-\rho} \frac{U \sqrt{D(l)}}{g d}\left(\frac{C_{d} a}{y_{s e}}\right)^{1 / 3}=f\left(\frac{d}{\eta}\right)
$$

Equation (14) can be used as a predictive formula for $y_{s e}$ if $f\left(\frac{d}{\eta}\right)$ is known.

\section{Empirical test}

The theoretical framework depicted in section 2 is now tested and further assessed against experimental data. The first thing to assess is to what extent viscous effects actually contribute to $\tau$ as a function of the flow regime, i.e. $d / \eta$. This is nicely captured by the ratio $u_{l} / u_{d}$, which essentially quantifies the ratio between $\tau$ estimated by including viscous effects (i.e. Equation 12) and $\tau$ estimated by assuming fully rough conditions (i.e. Equation 2). Figure 1(a) reports $u_{l} / u_{d}$ vs $d / \eta$ as estimated using the experimental data in $[6,12]$ assuming $b=5$. This dataset was chosen as it spans a wide range of $d / \eta$. Figure 1 (a) shows that, for $2<$ $\frac{d}{\eta}<78, u_{l} / u_{d}$ varies of about $5 \%$, meaning that, within this range, viscous effects on $\tau$ can be reasonably neglected. This allows Equation (14) to be solved explicitly for $y_{s e}$ as

$$
\frac{y_{s e} g}{U^{2}}=\frac{\frac{\rho}{\rho_{S}-\rho} C_{d}^{2 / 3}\left(\frac{a}{d}\right)^{2 / 3}}{f\left(\frac{d}{\eta}\right)}
$$

which is essentially the solution pertaining to the fully-rough case (i.e., Equation 6) modulated by the function $f\left(\frac{d}{\eta}\right)$.

The experimental data in $[6,12],[13]$ and [14] are now exploited to empirically derive the function $f\left(\frac{d}{\eta}\right)$ via Equation (15). This experimental dataset refers to cylindrical piers with circular cross section, uniform non-cohesive sediments, steady flows and large $B / a$ ratios (more details about the experimental data can be found in MB15). Figures 1(b), (c) and (d) show that, with exception of very few outliers, $f\left(\frac{d}{\eta}\right)$ nicely resembles a curve "a la Shields" as it displays a minimum that divides the so-called transitionally-smooth and the transitionally-rough branch. To guide the eye of the reader, the solid curves in figure 1 represent the Brownlie equation ([15]), which represents a good fit of the original Shields data. This function is vertically shifted by a factor $F_{1}$ that allows the curve to be within the same range as the experimental points. The dashed curve represents a reasonable fit of experimental data that was obtained by an ad hoc tuning of the coefficients appearing in the Brownlie equation. Interestingly, the main difference between the standard Brownlie curve and the proposed fit is a steeper rise of the branch in the transitionally-rough range (i.e. $d / \eta>$ 30). The reason for such a discrepancy is not entirely clear and is still matter of scrutiny by the authors. 
The dashed curves in figure 1 may be used for engineering applications and were obtained by the following function

$$
f=F_{1}\left\{0.22\left(\frac{d}{\eta}\right)^{-0.6}+0.09 e^{\left[-17.77\left(\frac{d}{\eta}\right)^{-0.6}\right]}\right\},
$$

where $F_{1}=25$ in panel (b), $F_{1}=60$ in panel (c) and $F_{1}=35$ in panel (d). Such variations in $F_{1}$ are due to the different definitions of equilibrium scour depth adopted by the authors of the three references where the experimental data were taken from (see MB15 for a full discussion on this aspect).

(a)

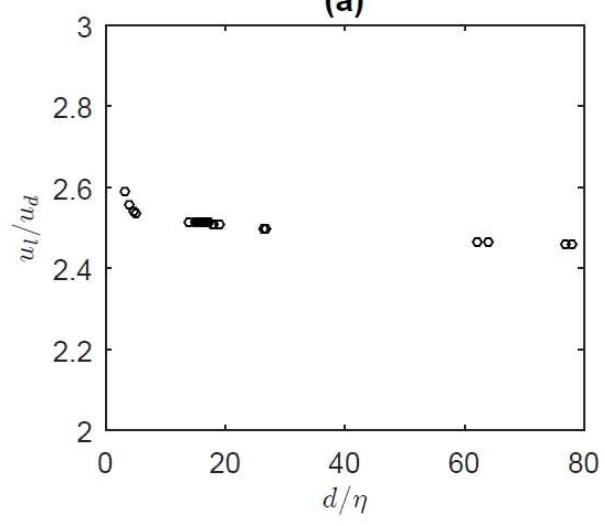

(c)

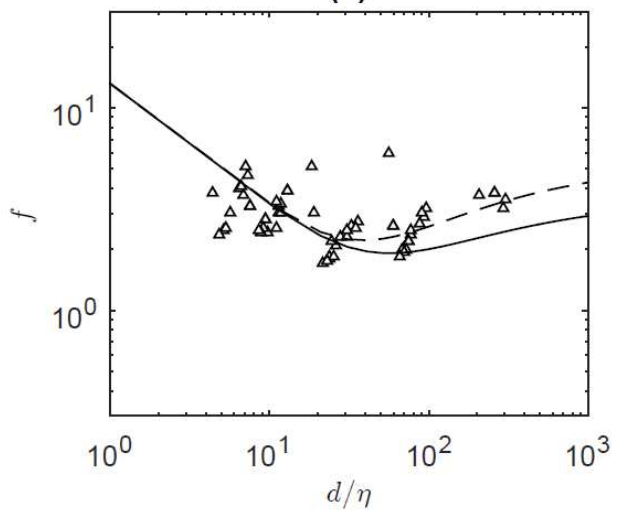

(b)

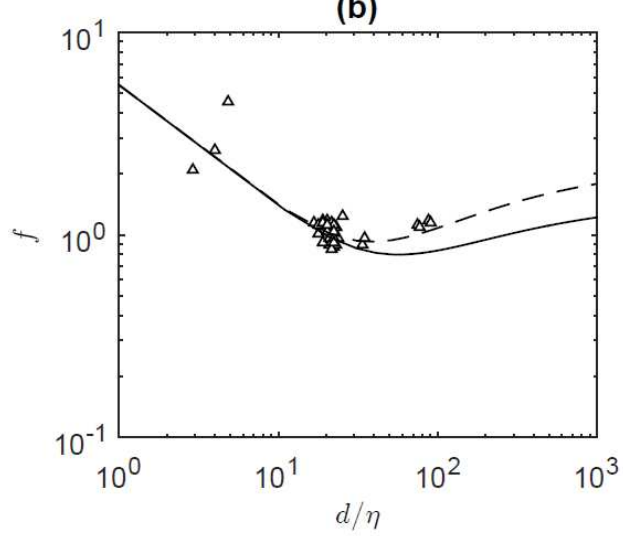

(d)

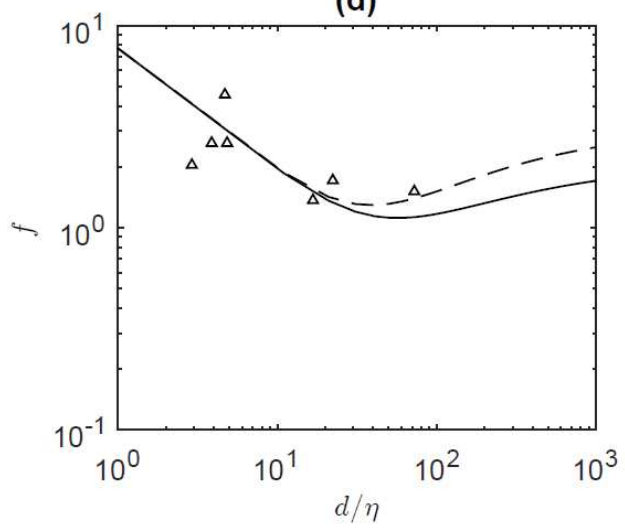

Figure 1. (a) $u_{l} / u_{d}$ vs $d / \eta$ estimated from the data by Lança et al. (2013) and (2015); panels (b)-(d) report the function $f$ (estimated using Equation 15) vs $d / \eta$ : (b) data from Lança et al. (2013) and (2015); (c) data from Ettema (1980); (d) data from Sheppard et al. (2006).

\section{Conclusions}

The aim of this paper was to provide a quantitative analysis of viscosity effects on equilibrium scour depths developing under clear-water conditions and around cylindrical structures. Towards this end the theoretical framework developed in a recent paper by Manes 
and Brocchini ([1]), initially based on the assumption of fully rough conditions, was extended to include such viscosity effects. This was done by assuming that near-wall eddies responsible for momentum transport have a characteristic size captured by the sum of the sediment diameter and the thickness of the viscous sub-layer, which was quantified by a bulk Kolmogorov length scale. The characteristic velocity of such eddies was estimated from the Batchelor structure function. Viscosity effects were also assumed to influence the critical shear stress at equilibrium conditions through a function $f(d / \eta)$, which was empirically derived using data taken from the literature. Interestingly, the shape of $f(d / \eta)$ closely resembles the standard Shields curve. An analytical formulation of $f(d / \eta)$ based on an ad hoc tuning of the Brownlie function is provided for use in engineering applications.

\section{Acknowledgements}

C. Manes acknowledges support from "Compagnia di San Paolo" (project: "Attrarre Docenti di qualità tramite Starting Grant").

\section{References}

1. C. Manes, M. Brocchini, J. Fluid Mech., 779, 27-49 (2015)

2. E. Tubaldi, L. Macorini, B.A. Izzuddin, C. Manes, F. Laio, Structural Safety 69, 11-22 (2017)

3. R. Ettema, G. Constantinescu, B. Melville, Evaluation of bridge scour research: pier scour processes and predictions. NCHRP Rep. 175 (2011)

4. B. Melville, S. Coleman, Bridge Scour, Water Resources Publications (2000)

5. R. Ettema, G. Kirkil, M. Muste, J. Hydraul. Eng. 132 (1), 33-40 (2006)

6. R. M. Lança, C. S. Fael, R. J. Maia, J. P. Pego, A. H. Cardoso, J. Hydraul. Eng. 139(11), 1117-1125 (2013)

7. H. W. Shen, V. R. Schneider, S. S. Karaki, J. Hydraul. Div., 95 (HY6), 1919-1940 (1969)

8. G. Nicollet, M. Ramette, Deformation des lits alluvionnaires affouillements autour des piles de ponts cylindriques. (Laboratoire Nacional d'Hydraulique, Chatou,1971) (in French).

9. J. M. Buffington, D. R. Montgomery, Water Resour. Res. 33 (8), 1993-2029 (1997)

10. D. Lohse, A.M. Groeling, Phys. Rev. Lett., 74 (10), 1747 (1995)

11. G. Gioia, P. Chakraborty, Phys. Rev. Lett., 96 (4), 044502 (2006)

12. R. M. Lança, G. Simarro, C. M. S. Fael, A. H. Cardoso,. J. Hydraul. Eng. 143 (3), 06015022 (2015)

13. R. Ettema, Scour at bridge piers. Rep. 216 (1980)

14. D. M. Sheppard, W. Jr. Miller, J. Hydraul. Eng, 132 (7), 635-642 (2006)

15. W. R. Brownlie, Prediction of flow depth and sediment discharge in open channels, (1981). 